\title{
OPEN Jasmonate resistant 1 and ethylene responsive factor 11 are involved in chilling sensitivity in pepper fruit (Capsicum annuUm L.)
}

\author{
Jeong Gu Lee ${ }^{1}$, Jieun Seo ${ }^{1}$, Byoung-Cheorl Kang ${ }^{1,2}$, Jeong Hee Choi ${ }^{3}$ \& Eun Jin Lee Le $^{1,4}$
}

Pepper fruit (Capsicum annuum L.) is sensitive to chilling stress with chilling injuries occurring below $7^{\circ} \mathrm{C}$; however, chilling injuries occur at different temperatures depending on the genotype. The present study aimed to identify the factors that affect chilling sensitivity in pepper fruits. A total of $112 F_{2}$ pepper fruits crossed between chilling-insensitive 'UZB-GJG-1999-51' and chilling-sensitive 'C00562' pepper were grouped according to the seed browning rate, which is a typical chilling symptom of pepper fruit under chilling conditions. Physiological traits, amino acids, fatty acids, as well as ethylene responsive factor (ERF) and jasmonate resistant 1 (JAR1) expression levels were analyzed, and their correlations with the seed browning rate were confirmed. The expression level of $J A R 1$ showed a strong negative correlation with the seed browning rate $(r=-0.7996)$. The expression level of ERF11 and content of hydrogen peroxide showed strong positive correlation with the seed browning rate ( $r=0.7622$ and 0.6607 , respectively). From these results, we inferred that JAR1 and ERF11 are important factors influencing the chilling sensitivity of pepper fruit.

Pepper (Capsicum annuum L.) is a subtropical vegetable that is sensitive to chilling stress. Thus, when subjected to chilling stress below $7^{\circ} \mathrm{C}$, various symptoms of chilling injury appear depending on the genotype, ripening stage, and treatment period of chilling ${ }^{1}$. In pepper fruit, the most common symptom of chilling injury is seed browning, which leads to reduced quality and economic loss after harvest. One of the main causes of seed browning is enzymatic browning by phenolic compounds. Total phenol content is associated to the oxidative browning of pepper seeds, and it has been reported that seed browning, induced by chilling stress, is related to polyphenol oxidase (PPO) and phenylalanine ammonia lyase $(\mathrm{PAL})^{2}$. Phenolic compounds are biosynthesized from phenylalanine, the precursor of phenolic compounds, by PAL, and the generated phenolic compounds are then oxidized to quinone by PPO. Finally, a brownish pigment is formed, and tissue browning occurs ${ }^{3}$.

Other major causes of seed browning are membrane peroxidation and increased reactive oxygen species (ROS), such as hydrogen peroxide, in embryos ${ }^{4}$. Under chilling stress conditions, cell membranes undergo enzymatic or non-enzymatic peroxidation in which unsaturated fatty acids are oxidized to saturated fatty acids, resulting in ROS production ${ }^{5}$. The generated ROS, in turn, react with the cell membrane to promote cell membrane peroxidation. To suppress these cycles, jasmonic acid (JA) signaling is activated, regulating the concentration of metabolites, such as amino acids and sugars, or inhibiting ROS production through the regulation of genes involved in ROS scavenging.

JA is one of the plant hormones, synthesized from $\alpha$-linolenic acid, which regulates plant growth and development extensively. In addition, JA plays an important role in response to herbivorous insects and to environmental stress $^{6}$, also is involved in chilling response enhancing chilling tolerance ${ }^{7,8}$. In addition, JA accumulation under chilling stress has been reported in Arabidopsis ${ }^{9}$, tomatoes ${ }^{10}$, and pepper ${ }^{11}$. JA should be conjugated with isoleucine by jasmonate resistant 1 (JAR1) to form jasmonoyl-isoleucine (JA-Ile) ${ }^{12,13}$. Then the generated JA-Ile binds to CORONATINE INSENSITIVE 1 to promote the ubiquitination of the JASMONATE-ZIM-domain protein, which acts as a repressor of JA signaling ${ }^{14}$.

\footnotetext{
${ }^{1}$ Department of Agriculture, Forestry and Bioresources, College of Agriculture and Life Sciences, Seoul National University, Seoul 08826, Republic of Korea. ${ }^{2}$ Plant Genomics and Breeding Institute, Seoul National University, Seoul 08826, Republic of Korea. ${ }^{3}$ Korea Food Research Institute, Wanju-gun, Jeollabuk-do 55365, Republic of Korea. ${ }^{4}$ Research Institute of Agriculture and Life Sciences, Seoul National University, Seoul 08826, Republic of Korea. ${ }^{\square}$ email: ejinlee3@snu.ac.kr
} 
Representative genes regulated by JA signaling are in the ethylene responsive factor (ERF) family. The ERF family contains AP2/ERF-type binding domains and is composed of the ERF and dehydration responsive element binding factor (DREB) subfamily ${ }^{15}$. The ERF family is involved in abiotic stress in a variety of plants, and the ERF subfamily, including ERFs 1, 2, 5, 6, and 11, is mainly involved in osmotic stress ${ }^{16}$. In contrast, the DREB subfamily is known to be involved in chilling stress response in Arabidopsis ${ }^{17,18}$. In pepper, CaPTI1 belonging to the ERF subfamily is involved in cold and drought stress and in Phytophthora capsici infection ${ }^{19}$.

However, previous research on chilling stress response in peppers is insufficient because the response and sensitivity to chilling stress differ depending on the pepper genotype. In our previous study, we confirmed the factors affecting the chilling sensitivity of pepper fruits ${ }^{20}$. When the chilling-insensitive 'UZB-GJG-1999-51' and chilling-sensitive 'C00562' peppers were exposed to chilling treatment at $2{ }^{\circ} \mathrm{C}$ for $24 \mathrm{~h}$, the expression levels of CaJAR1, CaERF 1, 3, 5, and 10 increased in both the genotypes. It was also confirmed that the expression levels of CaERF11 and CaDERB3 decreased in both the genotypes. In addition, the expression levels of CaJAR1, CaERF $1,3,5$, and 10 were higher in chilling-insensitive 'UZB-GJG-1999-51' pepper, and those of CaERF11 and CaD$R E B 3$ were higher in chilling-sensitive ' $C 00562$ ' pepper. As mentioned previously, JAR1 is a major activator of JA signaling through JA-Ile synthesis ${ }^{12,13}$. In addition, $E R F 1,3$, and 5 were reported to be upregulated under chilling stress in Arabidopsis ${ }^{21}$ and cotton ${ }^{22}$, and ERF10 has been shown to improve chilling tolerance in bananas ${ }^{23}$. ERF11 is known to promote plant internode elongation by activating the synthesis of gibberellin. In general, under chilling stress, plants respond through growth retardation ${ }^{24,25}$. Therefore, ERF11 seems to have a function of weakening the chilling tolerance. On this basis, in the chilling stress response, each of the former genes was expected to be a positive regulator, and the latter genes were expected to be candidate negative regulator genes.

In the present study, we investigated the factors that influence chilling sensitivity and seed browning in various genotypes of pepper fruit by confirming the correlations between the aforementioned candidate genes, ROS content, fatty acids, amino acids, and seed browning rate in pepper fruit.

\section{Material and methods}

Plant materials. We obtained pepper seeds of chilling-insensitive 'UZB-GJG-1999-51' and chilling-sensitive 'C00562' from Plant Genomics and Breeding Institute (Seoul National University, Seoul, Republic of Korea). The collection of 'UZB-GJG-1999-51' and 'C00562' peppers was permitted by Plant Genomics and Breeding Institute and complies with relevant institutional, national, and international guidelines and legislation. A total of $112 \mathrm{~F}_{2}$ pepper fruits obtained by crossing ' $U Z B-G J G-1999-51$ ' and 'C00562' pepper were harvested 45-50 days after full bloom in a greenhouse at Seoul National University (Suwon, Republic of Korea). Immediately after harvest, they were precooled at $18{ }^{\circ} \mathrm{C}$ for $8 \mathrm{~h}$, and then the pepper fruits were transferred to $2{ }^{\circ} \mathrm{C}$ for chilling treatment. After chilling treatment for 3 weeks, each individual fruit was cut lengthwise into half and seed browning was observed and photographed. The seed browning rate of each fruit was calculated using the following equation ${ }^{20}$ :

$$
\text { Seed browning rate }(\%)=\frac{\text { the number of browned seeds }}{\text { the number of total }(\text { normal }+ \text { browned }) \text { seeds }} \times 100
$$

For further analysis, we grouped at $10 \%$ intervals using the seed browning rate for $\mathrm{F}_{2}$ fruits. The groups were designated as Group 1 through Group 7, with seed browning rates being 0-10\%, 10-20\%, 20-30\%, 30-40\%, $40-50 \%, 50-60 \%$, and more than $60 \%$, respectively (Fig. 1 and Supplementary Table 1). In addition, both ' $U Z B$ GJG-1999-51' and 'C00562' fruits were treated at a chilling temperature of $2{ }^{\circ} \mathrm{C}$ for 3 weeks. All the seeds of each fruit were collected without placenta, immediately frozen in liquid nitrogen, and stored at $-80{ }^{\circ} \mathrm{C}$ for all experiments.

Hydrogen peroxide content analysis. The hydrogen peroxide content was analyzed following a previously described method ${ }^{26}$ with slight modifications. First, frozen pepper seeds were completely ground into a fine powder using a mortar and pestle in liquid nitrogen. Then, $100 \mathrm{mg}$ of frozen pepper seed powder was combined with $1 \mathrm{~mL}$ cold $\left(4^{\circ} \mathrm{C}\right)$ trichloroacetic acid $\left(0.1 \%\right.$, w/v), and incubated at $4{ }^{\circ} \mathrm{C}$ for $10 \mathrm{~min}$. All samples were centrifuged at $12,000 \times g$ at $4^{\circ} \mathrm{C}$ for $20 \mathrm{~min}$. Each $0.5 \mathrm{~mL}$ supernatant was mixed with $0.5 \mathrm{~mL}$ of $1 \mathrm{M} \mathrm{KI}$ and $0.25 \mathrm{~mL}$ of $10 \mathrm{mM}$ potassium phosphate buffer ( $\mathrm{pH}$ 7.0). The mixtures were incubated in the dark at $22^{\circ} \mathrm{C}$ for $20 \mathrm{~min}$. Absorbance was measured at $390 \mathrm{~nm}$ using a microplate spectrophotometer (Biotek Epoch, Winooski, VT, USA). A standard curve was plotted using 0, 3.675, 7.35, 14.7, and $29.4 \mathrm{mM}$ of hydrogen peroxide solutions.

Total phenolic content and total antioxidant activity analysis. First, $200 \mathrm{mg}$ of frozen pepper seed powder was combined with $10 \mathrm{~mL}$ of $80 \%(\mathrm{v} / \mathrm{v})$ methanol, sonicated for $20 \mathrm{~min}$, and centrifuged at $3,000 \times g$ at $22^{\circ} \mathrm{C}$ for $20 \mathrm{~min}$. Then, each supernatant was transferred to a new $15 \mathrm{~mL}$ tube for total phenolic content and total antioxidant activity assays.

Total phenolic content was determined using the Folin-Ciocalteu reagent ${ }^{27}$. First, $50 \mu \mathrm{L}$ of diluted solution and $50 \mu \mathrm{L}$ Folin-Ciocalteu reagent were added to $450 \mu \mathrm{L}$ of distilled water. The mixture was vortexed briefly and incubated at $22{ }^{\circ} \mathrm{C}$ for $5 \mathrm{~min}$. Then, $150 \mu \mathrm{L}$ of $20 \%(\mathrm{w} / \mathrm{v}) \mathrm{Na}_{2} \mathrm{CO}_{3}$ and $200 \mu \mathrm{L}$ distilled water were added, and the mixture was incubated in the dark at $22^{\circ} \mathrm{C}$ for $30 \mathrm{~min}$. Absorbance was measured at $750 \mathrm{~nm}$ using a microplate spectrophotometer (Biotek Epoch). A standard curve was plotted using 0, 12.5, 25, 50, 100, and $200 \mathrm{mg} \mathrm{L}^{-1}$ gallic acid solutions.

Total antioxidant activity was determined by evaluating the ABTS $\bullet{ }^{+}$scavenging activity ${ }^{28}$. The ABTS $\bullet^{+}$solution was diluted with distilled water to obtain an $\mathrm{OD}_{734}=0.7$. Then, $1.2 \mathrm{~mL}$ diluted ABTS $\bullet^{+}$solution was mixed with $10 \mu \mathrm{L}$ of the sample. The mixture was vortexed briefly and incubated in the dark at $22{ }^{\circ} \mathrm{C}$ for $15 \mathrm{~min}$. 


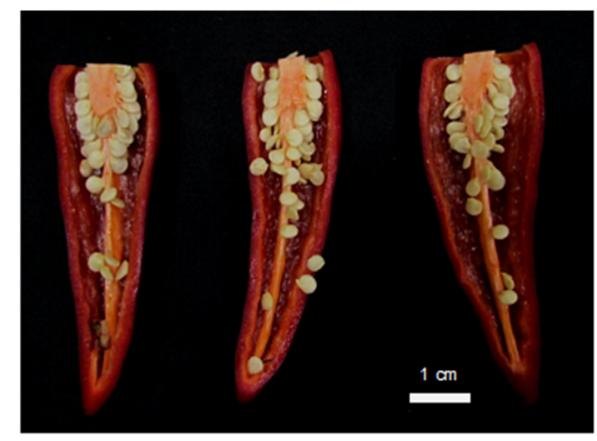

'UZB-GJG-1999-51'

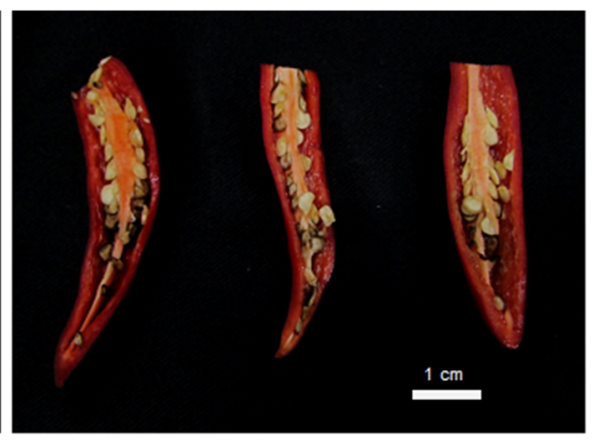

'C00562'

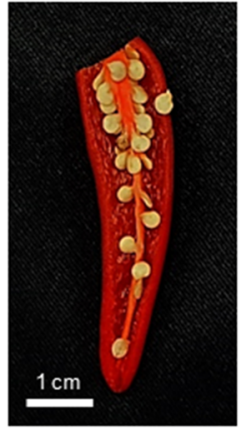

Group 1

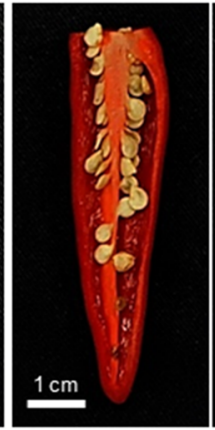

Group 2

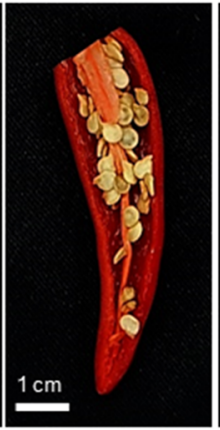

Group 3

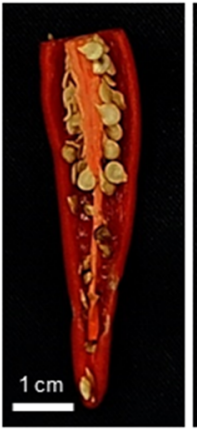

Group 4

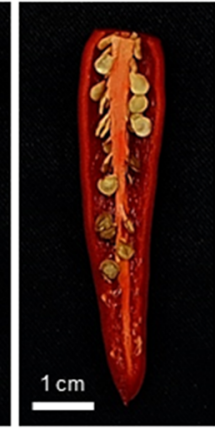

Group 5

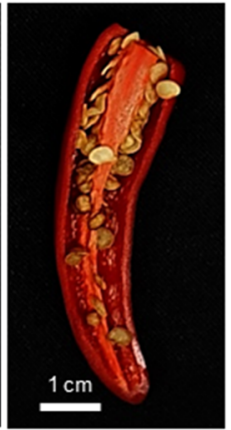

Group 6

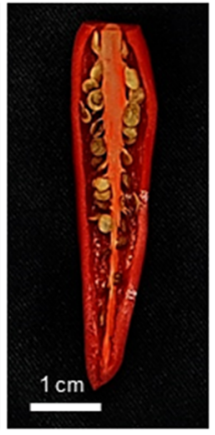

Group 7

Figure 1. Seed browning appearances of 'UZB-GJG-1999-51', 'C00562' (upper pictures), and $\mathrm{F}_{2}$ groups (bottom pictures) after chilling at $2{ }^{\circ} \mathrm{C}$ for 3 weeks. The groups were designated as Group 1 through Group 7, with seed browning rates being $0-10 \%, 10-20 \%, 20-30 \%, 30-40 \%, 40-50 \%, 50-60 \%$, and $>60 \%$, respectively. The pictures in the upper panel were published as "Jasmonic acid and ERF family genes are involved in chilling sensitivity and seed browning of pepper fruit after harvest" by J. G. Lee, G. Yi, J. Seo, B. C. Kang, J. H. Choi, and E. J. Lee, 2020, in Scientific Reports, volume 10, Results section, Fig. 1. CC BY.

Absorbance was measured at $734 \mathrm{~nm}$ using a microplate spectrophotometer (Biotek Epoch). A standard curve was plotted using $0,0.1,0.2,0.4,0.8$, and $1.6 \mathrm{mM}$ Trolox solutions.

Free amino acid analysis by high-performance liquid chromatography (HPLC). Free amino acids were analyzed following a previously described method ${ }^{29}$ with some modifications. First, $1.2 \mathrm{~mL}$ of $5 \%$ trichloroacetic acid was added to $100 \mathrm{mg}$ of frozen pepper seed powder, and the mixture was sonicated at $22{ }^{\circ} \mathrm{C}$ for $30 \mathrm{~min}$. After centrifugation at $12,000 \times \mathrm{g}$ at $4{ }^{\circ} \mathrm{C}$ for $20 \mathrm{~min}, 1 \mathrm{~mL}$ of the supernatant was collected and filtered through a $0.45 \mu \mathrm{m}$ polyvinylidene fluoride membrane filter. After mixing $5 \mu \mathrm{L}$ of $0.4 \mathrm{~N}$ borate buffer ( $\mathrm{pH}$ 10.2 ) and $1 \mu \mathrm{L}$ of sample, $1 \mu \mathrm{L}$ of o-phthalaldehyde and $1 \mu \mathrm{L}$ of fluorenylmethyloxycarbonyl were added for derivatization. Finally, $64 \mu \mathrm{L}$ of distilled water was added, and the mixture was analyzed by HPLC. The column was equipped with a Zorbax Eclipse AAA $(4.6 \times 150 \mathrm{~mm}$, Agilent, Santa Clara, CA, USA), and the flow rate was set to $2 \mathrm{~mL} \cdot \mathrm{min}^{-}{ }^{1}$. Mobile phase A was set to $40 \mathrm{mM} \mathrm{NaH}_{2} \mathrm{PO}_{4}(\mathrm{pH} 7.8)$, and $\mathrm{B}$ was set to acetonitrile:methanol: $\mathrm{H}_{2} \mathrm{O}$ (45:45:10, v:v:v).

Fatty acid analysis by gas chromatography (GC). Fatty acids were analyzed following a previously described method ${ }^{30}$ with some modifications. First, $100 \mathrm{mg}$ of ground frozen pepper seed was placed in a Teflon-cap tube and extracted with $2 \mathrm{~mL}$ of methylation mixture (methanol:benzene:2,2'dimethoxypropane: $\mathrm{H}_{2} \mathrm{SO}_{4}=39: 20: 5: 2, \mathrm{v}: \mathrm{v}: \mathrm{v}: \mathrm{v}$ ) and $1 \mathrm{~mL}$ of heptane at $80{ }^{\circ} \mathrm{C}$ for $2 \mathrm{~h}$. After cooling to $22{ }^{\circ} \mathrm{C}$, the supernatant was collected and analyzed through GC using the Agilent 7890A system (Agilent). The column was DB-23 $\left(0.25 \times 60 \times 0.25 \mu \mathrm{m}\right.$, Agilent). The flame ionization detector (FID) was set at $280{ }^{\circ} \mathrm{C}$, and the flow rates were $35 \mathrm{~mL} \cdot \mathrm{min}^{-1}$ for $\mathrm{H}_{2}, 350 \mathrm{~mL} \cdot \mathrm{min}^{-1}$ for air, and $35 \mathrm{~mL} \cdot \mathrm{min}^{-1}$ for He. The injector temperature was $250^{\circ} \mathrm{C}$. The oven temperature was increased from $50^{\circ} \mathrm{C}$ to $130^{\circ} \mathrm{C}$ at $15^{\circ} \mathrm{C} \cdot \mathrm{min}^{-1}, 170^{\circ} \mathrm{C}$ by $8{ }^{\circ} \mathrm{C} \cdot \mathrm{min}^{-1}$, and $215^{\circ} \mathrm{C}$ by $2{ }^{\circ} \mathrm{C} \cdot \mathrm{min}^{-1}$.

Total RNA extraction and cDNA synthesis. Frozen seeds were ground into a fine powder using a mortar and pestle in liquid nitrogen, and $100 \mathrm{mg}$ powder was used for total RNA extraction using the Ribospin Seed/ Fruit Kit (Geneall, Seoul, South Korea) following the manufacturer's instructions. The extracted total RNA was used for cDNA synthesis. cDNA was synthesized using an amfiRivert Platinum cDNA Systhesis Master Mix Kit (Gendepot, Baker, TX, USA) following the manufacturer's instructions. 
Quantitative PCR (qPCR) analysis. The cDNA was diluted to $50 \mathrm{ng} \mu \mathrm{L}^{-1}$, and $\mathrm{qPCR}$ was performed using the $2 \times$ Real-Time PCR Master Mix (Biofact, Daejeon, South Korea) in a final volume of $20 \mu \mathrm{L}$ and CFX Connect Real-Time System (Bio-rad, Hercules, CA, USA) under the following conditions: $95{ }^{\circ} \mathrm{C}$ for $15 \mathrm{~min}$, followed by 40 cycles at $95^{\circ} \mathrm{C}$ for $20 \mathrm{~s}, 55^{\circ} \mathrm{C}$ for $40 \mathrm{~s}$, and $72{ }^{\circ} \mathrm{C}$ for $20 \mathrm{~s}$. Relative expression was determined by normalization against the expression of the pepper Actin7. The primers used for qPCR are listed in Supplementary Table 2 and were designed based on the reference gene set using Primer 3 plus (http://www.bioinforma tics.nl/cgi-bin/primer3plus/primer3plus.cgi). Relative gene expression was calculated using the $2^{-\Delta \Delta C t} \operatorname{method}^{31}$.

Statistical analysis. The experiments were conducted in a randomized design with three replicates of 'UZB-GJG-1999-51' and 'C00562' and five replicates of $\mathrm{F}_{2}$ groups. Statistical comparisons between the means of the experimental groups were performed using SPSS ver. 26.0 (IBM Corp., Armonk, NY, USA). One-way analysis of variance with Duncan's multiple range test was performed to determine significant differences. The metabolite data were auto-scaled and used for heat-map analysis and Pearson's correlation analysis in MetaboAnalyst 5.0 (www.metaboanalyst.ca) and correlation network analysis in Cytoscape v3.6.1 (http://cytoscape.github.io/).

\section{Results}

Seed browning rates of various pepper genotypes. After chilling at $2{ }^{\circ} \mathrm{C}$ for 3 weeks, there were significant differences in the seed browning rate between each $\mathrm{F}_{2}$ pepper fruit. We screened $112 \mathrm{~F}_{2}$ individuals, as well as ' $U Z B-G J G-1999-51$ ' and 'C00562' pepper fruit before and after chilling treatment (Fig. 1 and Supplementary Table 1). The seed browning rate of both ' $U Z B-G J G-1999-51$ ' and 'C00562' was $0 \%$ before chilling treatment and $0 \%$ and $62.99 \%$, respectively, after chilling treatment. The seed browning rate of the $\mathrm{F}_{2}$ individuals ranged from $0 \%$ to $77.42 \%$, and the average seed browning rate of groups 1 to 7 was $2.75 \%, 14.49 \%, 26.14 \%, 35.13 \%$, $44.57 \%, 53.37 \%$, and $66.61 \%$, respectively.

Hydrogen peroxide, total phenolic content, and total antioxidant activity. The changes in hydrogen peroxide content, total phenolic content, and total antioxidant activity were confirmed according to the seed browning rate (Fig. 2). Hydrogen peroxide content increased in both 'UZB-GJG-1999-51' and 'C00562' peppers after chilling at $2^{\circ} \mathrm{C}$. In addition, as the seed browning rate increased in the $\mathrm{F}_{2}$ individuals, the hydrogen peroxide content significantly increased in groups 6 and 7 when compared to groups 1 and 2 (Fig. 2A). There was no significant difference in total phenolic content before and after chilling in both 'UZB-GJG-1999-51' and 'C00562' peppers. Moreover, $\mathrm{F}_{2}$ individuals showed significantly higher content in groups 5 and 7 , but no significant difference was found in the other groups (Fig. 2B). Before or after chilling treatment, total antioxidant activity did not differ in 'UZB-GJG-1999-51' pepper, but was significantly decreased in 'C00562' pepper after chilling treatment. In $\mathrm{F}_{2}$ individuals, group 4 showed significantly higher activity, but there was no significant difference between the other groups (Fig. 2C).

Heat-map analysis of free amino acid and fatty acid content. The content of 20 amino acids and nine fatty acids was quantified by HPLC and GC-FID. To confirm the relationships between the seed browning rate and metabolites, a heat-map analysis was performed using changes in metabolites (Fig. 3). In ' $U Z B$ GJG-1999-51' pepper, fatty acid and amino acid content did not show an obvious change. These results might be because the seeds were less damaged by chilling treatment, and as a result, seed browning did not occur. Valine, isoleucine, and linoleic acid content slightly increased, and palmitic acid content slightly decreased. Conversely, in 'C00562' pepper, in which seed browning occurred following chilling treatment, the content of most amino acids, except for glutamic acid, increased. In addition, palmitic acid content increased, and linoleic acid content decreased. In $\mathrm{F}_{2}$ individuals, as the seed browning rate increased, the content of a number of amino acids, including branched-chain amino acids (BCAAs), GABA, and phenylalanine, tended to increase. However, aspartic acid, glutamic acid, asparagine, and glycine were not significantly correlated with the seed browning rate. In the case of fatty acids, palmitic acid, a saturated fatty acid, increased as the seed browning rate increased, whereas linoleic acid, an unsaturated fatty acid, decreased.

Relative gene expression analysis by qPCR. From the gene expression analysis of CaERF 1, 3, 5, and 10, selected as positive regulator candidate genes for chilling response in previous studies, there was no significant difference in relative gene expression levels before and after chilling in both 'UZB-GJG-1999-51' and 'C00562' peppers. Moreover, expression levels of CaERF 1, 3, 5, and 10 tended to be higher in 'UZB-GJG-1999-51' than in 'C00562', but there was no significant difference between the genotypes (Fig. 4 ). In the analysis of $\mathrm{F}_{2}$ individuals, CaERF1 expression levels were higher in groups 3 and 5 but not significantly. CaERF3 expression level was significantly higher in group 4 than in other groups except group 6. CaERF5 expression level was significantly higher in group 2 than in group 3. In addition, CaERF10 expression levels were significantly higher in groups 3 and 4 than in group 7, but there was no tendency to change according to the seed browning rate. However, in the case of CaJAR1, another candidate positive regulator gene, the expression level was significantly increased after chilling in 'UZB-GJG-1999-51' pepper. In addition, from the analysis between $\mathrm{F}_{2}$ individuals, it was confirmed that the expression level significantly decreased as the seed browning rate increased.

CaERF11 and CaDREB3, candidates for negative regulator genes in chilling response, showed similar gene expression trends. In both 'UZB-GJG-1999-51' and 'C00562' pepper, the expression level of CaERF11 and CaD$R E B 3$ significantly decreased after chilling treatment, and was higher in 'C00562' than in 'UZB-GJG-1999-51'. In the analysis of $\mathrm{F}_{2}$ individuals, the expression level of CaERF11 increased significantly with the increase in seed 


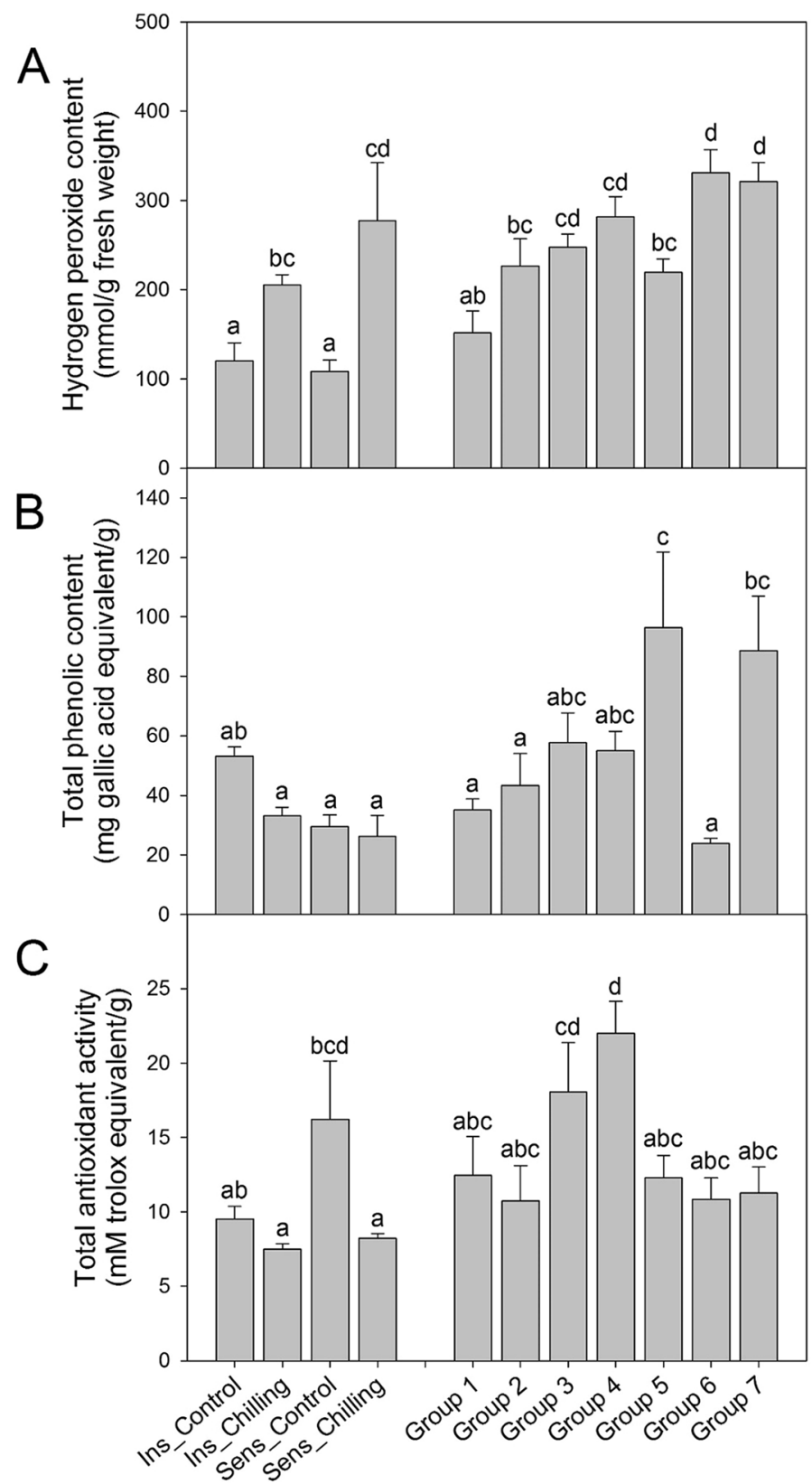

Figure 2. Changes in physiological traits of ' $U Z B-G J G-1999-51$ ', 'C00562', and $\mathrm{F}_{2}$ groups after chilling treatment at $2{ }^{\circ} \mathrm{C}$ for 3 weeks. (A) Hydrogen peroxide content, (B) total phenolic content, and (C) total antioxidant activity. Ins, chilling-insensitive 'UZB-GJG-1999-51' pepper; Sens, chilling-sensitive 'C00562' pepper. The groups were divided from Group 1 to Group 7, and each seed browning rate was 0-10\%, 10-20\%, 20-30\%, 30-40\%, 40-50\%, $50-60 \%$, and $>60 \%$, respectively. Vertical bars are means \pm SE. Different letters represent significant differences $(p<0.05)$ in Duncan's multiple range test.

browning rate, but $C A D R E B 3$ was significantly higher only in group 6, and there was no significant difference in the other groups.

Correlations between seed browning rate and analysis factors. To identify the factors involved in pepper seed browning, correlation analysis was performed using physiological traits, metabolites, and gene expression levels. First, the results of Pearson's correlation heat-map analysis confirmed that seed browning was positively correlated with CaERF11 expression level and hydrogen peroxide content at $\mathrm{r}=0.7622$ and 0.6607 , respectively (Fig. 5A). In contrast, it was confirmed that seed browning was negatively correlated with CaJAR1 expression level $(\mathrm{r}=-0.7996)$. Furthermore, CaJAR1 was positively correlated with CaERF10 expression level and total antioxidant activity. In addition, $C a E R F 1$ expression level was positively correlated with aspartic acid 


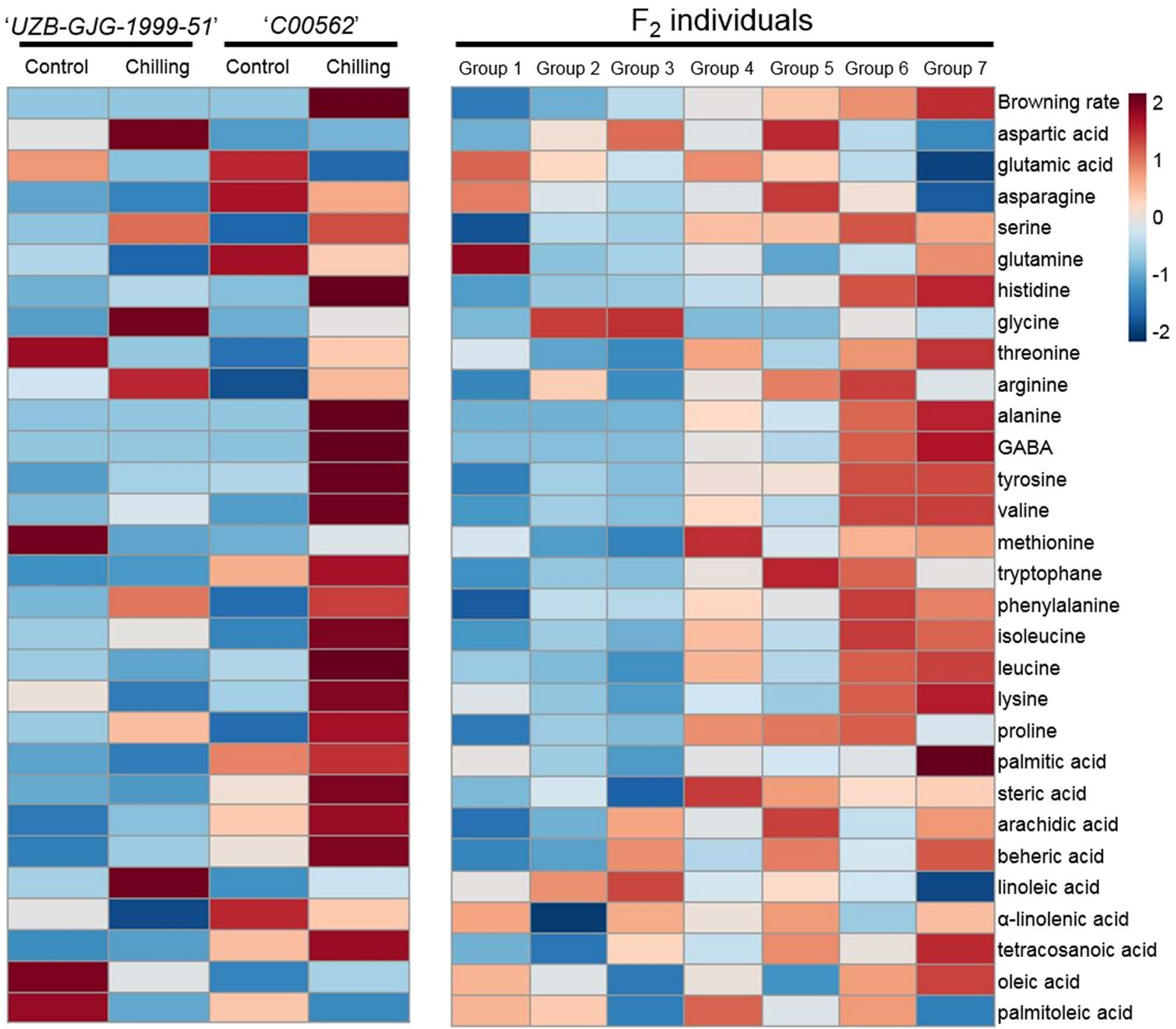

Figure 3. Heat map of free amino acids and fatty acids in ' $U Z B-G J G-1999-51$ ', 'C00562', and $\mathrm{F}_{2}$ groups after chilling treatment at $2{ }^{\circ} \mathrm{C}$ for 3 weeks. The groups were divided from Group 1 to Group 7, and each seed browning rate was $0-10 \%, 10-20 \%, 20-30 \%, 30-40 \%, 40-50 \%, 50-60 \%$, and $>60 \%$, respectively. The mean foldchange magnitude for overall metabolites in each group is color-coded. Increases are shown in red while blue indicates decreases. This figure was created with freely available MetaboAnalyst (version 5.0, https://www.metab oanalyst.ca/).

content and total phenolic content, and CaERF3 and CaERF5 expression levels were positively correlated with linoleic acid content.

To clearly visualize the correlation between seed browning and the analyzed factors, a correlation network was analyzed based on Pearson's correlation coefficient $(\geq|0.7|, p<0.05)$ (Fig. 5B). Through correlation network analysis, it was confirmed that CaJAR1 expression level was negatively correlated with the seed browning rate and CaERF11 expression level ( $\mathrm{r}=-0.7996$ and -0.7254 , respectively). In addition, isoleucine, leucine, and valine, belonging to BCAA, were positively correlated with each other. In terms of fatty acids, oleic acid was negatively correlated with aspartic acid $(\mathrm{r}=-0.7158)$, and palmitic acid and linoleic acid, and the representative saturated and unsaturated fatty acids, respectively, were negatively correlated $(\mathrm{r}=-0.9530)$.

CaJAR1 and CaERF11 expression levels and hydrogen peroxide content, which were correlated with the seed browning rate in the heat-map and network analyses, were used to confirm the individual correlation with the seed browning rate (Fig. 6). CaJAR1 expression level was confirmed as the coefficient of determination $\left(R^{2}\right)$ with a seed browning rate of $0.6393(p<0.001)$ (Fig. 6A). The $R^{2}$ for seed browning with CaERF11 expression level and hydrogen peroxide content were 0.5809 and 0.4365 , respectively (Fig. 6B,C). 


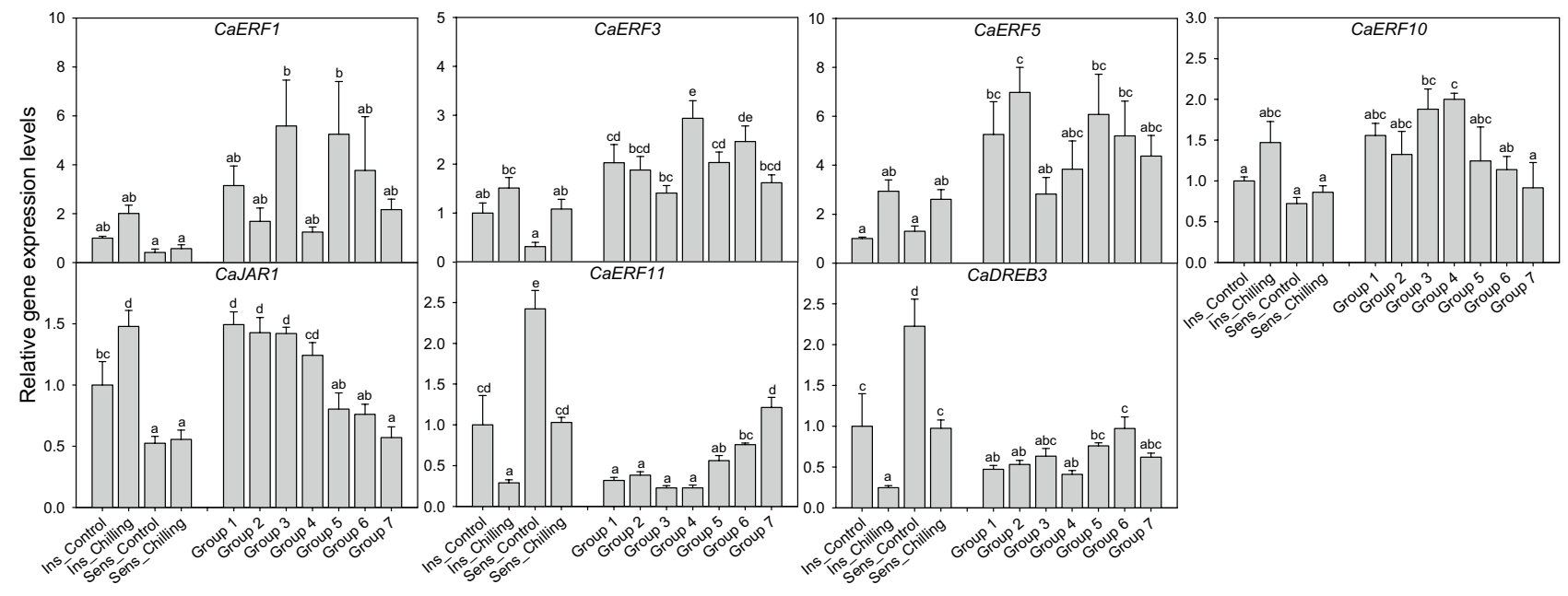

Figure 4. Relative expression levels of CaJAR1 and CaERF family genes in ' $U Z B-G J G-1999-51$ ', 'C00562', and $\mathrm{F}_{2}$ groups after chilling treatment at $2{ }^{\circ} \mathrm{C}$ for 3 weeks. Ins, chilling-insensitive 'UZB-GJG-1999-51' pepper; Sens, chilling-sensitive 'C00562' pepper. The groups were designated as Group 1 through Group 7, with seed browning rates being $0-10 \%, 10-20 \%, 20-30 \%, 30-40 \%, 40-50 \%, 50-60 \%$, and $>60 \%$, respectively. Vertical bars are means \pm SE. Different letters represent significant differences $(p<0.05)$ in Duncan's multiple range test.

\section{Discussion}

As a result of chilling, in $112 \mathrm{~F}_{2}$ individual pepper fruits obtained from crossing chilling-insensitive 'UZBGJG-1999-52' and chilling-sensitive 'C00562' peppers, the seed browning rate was widely distributed, ranging from 0 to $77.42 \%$ (Fig. 1). From these results, we inferred that the seed browning rate and chilling sensitivity of pepper are affected by genetic factors. To identify the factors influencing chilling sensitivity, $\mathrm{F}_{2}$ individuals were classified and grouped based on the seed browning rate, and physiological traits, metabolites, and gene expression levels were identified.

First, it was confirmed that the hydrogen peroxide content was significantly correlated with the browning rate $(\mathrm{r}=0.6607)$ and increased as the seed browning rate increased (Fig. 2A). Furthermore, previous studies reported that total phenol content and phenylalanine increased, showing a significant correlation with tissue browning during chilling stress ${ }^{32}$, while total phenol content was reported to decrease in pepper seeds during chilling treatment ${ }^{33}$. In the present study, total phenol content was not significantly correlated with seed browning after chilling (Fig. 2B). However, phenylalanine content was positively correlated with the seed browning rate $(\mathrm{r}=0.6120)$ (Figs. 3 and 5$)$.

After chilling treatment, total antioxidant activity was not significantly different between the groups, except for group 4 (Fig. 2). Also, in this study, the tendency of changes in the antioxidant activity and total phenol content associated with chilling sensitivity was not similar, and there was no significant difference. These results are not consistent with those of a previous study wherein changes in the antioxidant activity were observed to have a high correlation with the changes in the total phenol content ${ }^{34}$. Under abiotic stress, such as chilling, photochemical systems I and II are damaged, and the generated excitation energy is transferred to oxygen molecules to generate harmful ROS, such as hydrogen peroxide, $\mathrm{O}_{2}^{-}$, and $\mathrm{OH}^{-35}$. Plants have enzymatic and non-enzymatic antioxidant systems against ROS. Antioxidant enzymes include superoxide dismutase, catalase, ascorbate peroxidase, and glutathione-S-transferase ${ }^{36}$, and non-enzymatic antioxidant metabolites include ascorbic acid, glutathione, and phenolic compounds ${ }^{37}$. Total phenol content and antioxidant activity vary mainly based on genotypes and environmental factors, such as storage temperature and duration ${ }^{38,39}$. Even in previous studies, the changes in the total phenol content and antioxidant capacity were not consistent depending on the storage period ${ }^{38,40}$, probably because of various factors, such as genetics, ROS, and expression of related genes.

In the present study, palmitic acid, linoleic acid, GABA, leucine, valine, and isoleucine had a significant correlation with seed browning (Fig. 3). Palmitic acid and linoleic acid, which are saturated and unsaturated fatty acids, respectively, were negatively correlated with $r=-0.9530$ (Fig. 5). When plants are subjected to chilling stress, lipid peroxidation occurs, and unsaturated fatty acids are oxidized to saturated fatty acids. ROS, generated during this process, promotes chilling response signaling but accumulates cell damage within the plant ${ }^{41}$. Therefore, changes in the content of palmitic acid and linoleic acid in pepper seeds after chilling treatment serve as indicators of damage and ROS generation induced by chilling stress.

After chilling treatment, the change in GABA content was positively correlated with the seed browning rate $(\mathrm{r}=0.7402)$ (Figs. 3 and 5). GABA is involved in most abiotic stresses and is synthesized upon recognition of $\mathrm{Ca}^{2+}$ ions generated by damage in plants ${ }^{42}$. Therefore, it is expected that the GABA content will increase as the chilling stress damage increases.

Changes in the content of isoleucine, leucine, and valine were found to have correlation coefficients (r) of $0.5099,0.5688$, and 0.5674 , respectively, with the seed browning rate after chilling treatment. In addition, it can be seen that the correlation coefficient between each of the three amino acids was high from 0.8120 to 0.9595 (Fig. 5). These amino acids belong to BCAA, and they share a biosynthetic pathway with pyruvic acid ${ }^{43}$. 


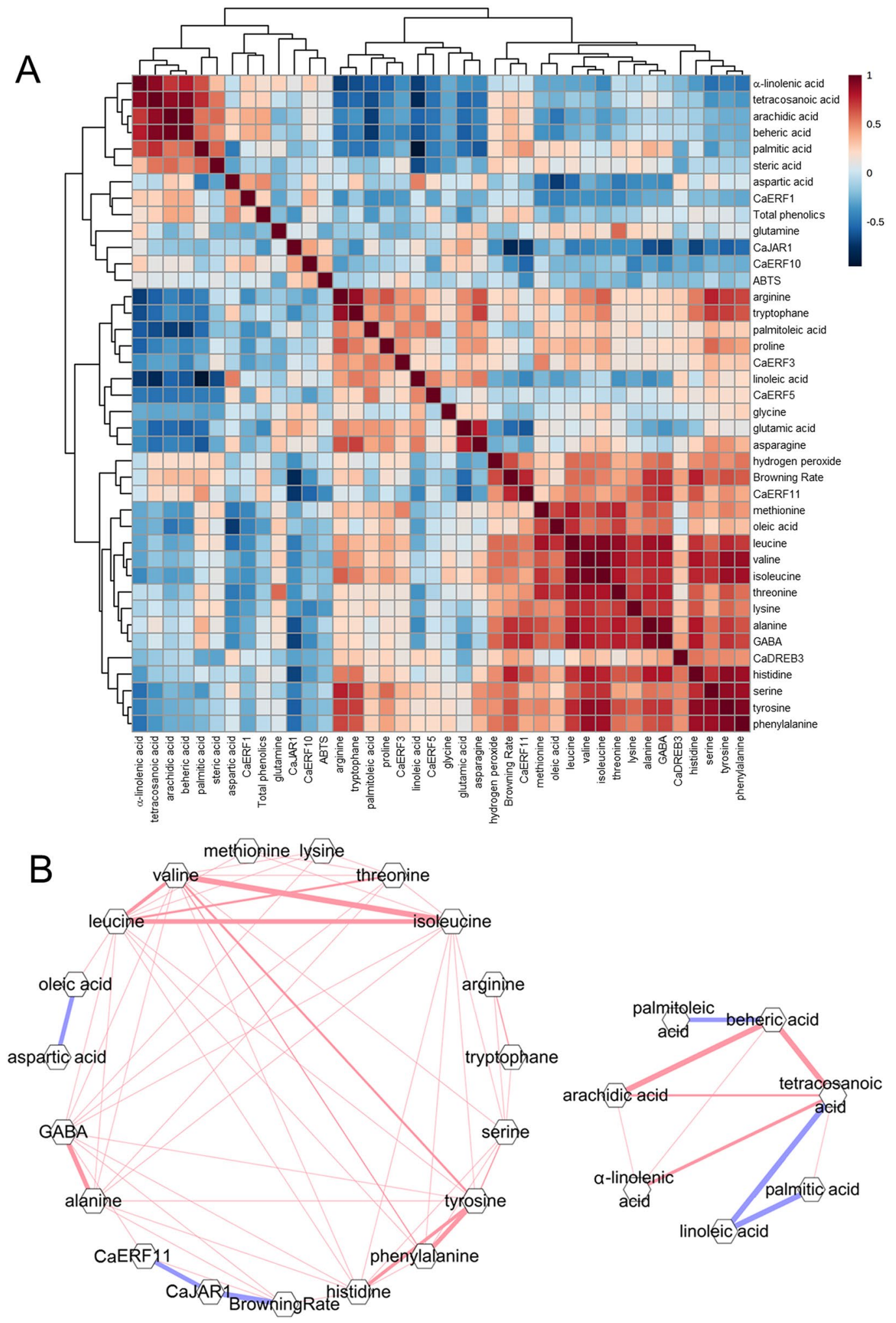

Figure 5. Correlation heat map (A) and networks (B) among physiological traits, metabolic compounds, and expression levels of CaJAR1 and CaERF family genes in 'UZB-GJG-1999-51', 'C00562', and $\mathrm{F}_{2}$ groups after chilling treatment at $2{ }^{\circ} \mathrm{C}$ for 3 weeks. Positive and negative correlations are shown as red and blue lines, respectively, with the line thickness corresponding to a higher or lower Pearson's correlation for correlation $\geq|0.70|$. Correlation heat map is created with freely available MetaboAnalyst (version 5.0, https:// www.metaboanalyst.ca/) and networks are created with Cytoscape software (version3.6.1, https://cytoscape. github.io/). 


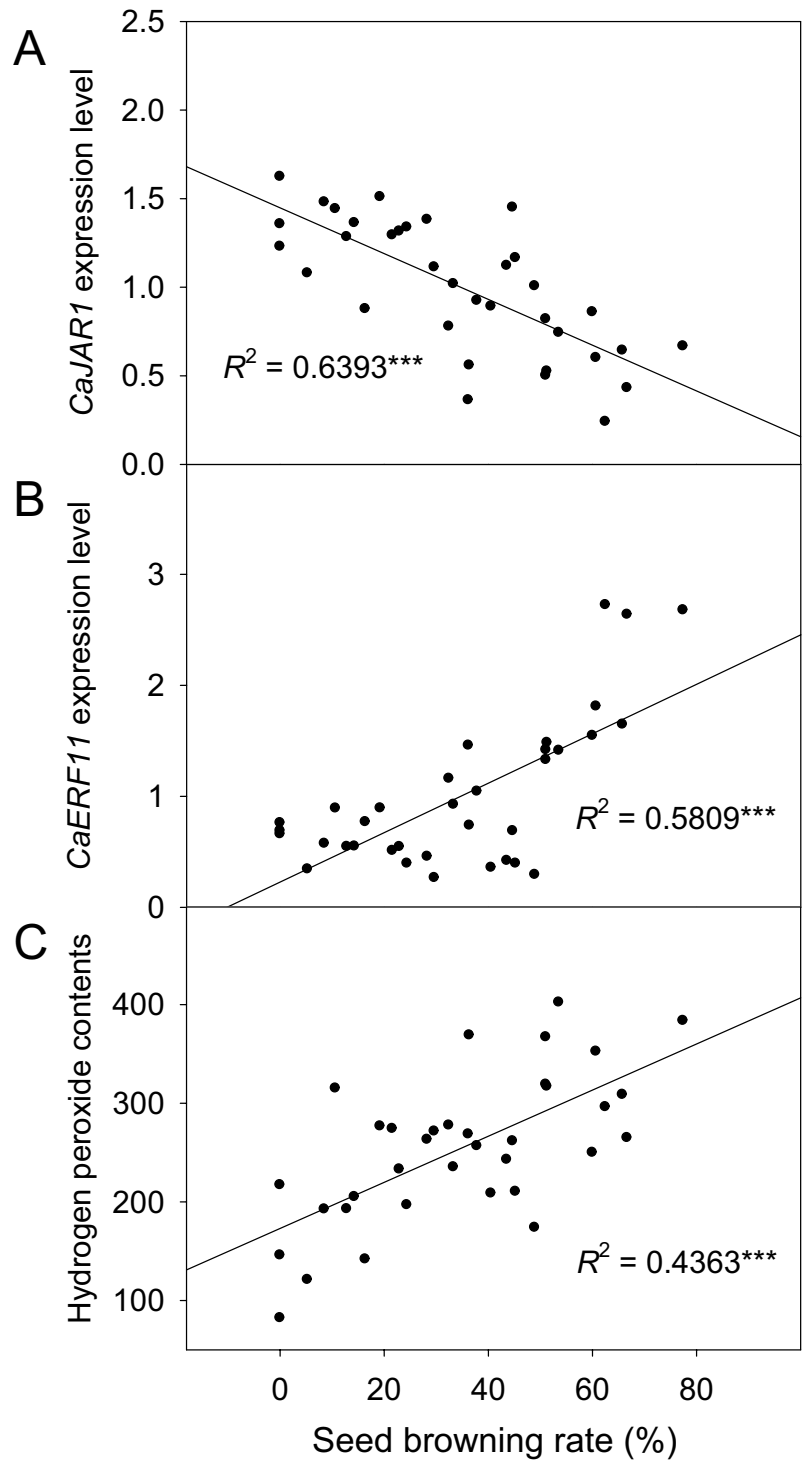

Figure 6. Correlation analysis of the seed browning rate with CaJAR1 expression level (A), CaERF11 expression level (B), and hydrogen peroxide content (C) in ' $U Z B-G J G-1999-51$ ', 'C00562', and $\mathrm{F}_{2}$ groups after chilling treatment at $2^{\circ} \mathrm{C}$ for 3 weeks.

In particular, isoleucine plays an important role in activating JA signaling by forming JA-Ile through JAR $1^{44}$. Previous studies have confirmed that the endogenous JA content of pepper fruits and seeds is low, and the content of isoleucine increases with increasing seed browning ${ }^{11}$. Therefore, we inferred that the chilling response mechanism of pepper under chilling stress is regulated according to the expression level of CaJAR1, a gene that synthesizes JA-Ile. In the present study, the expression level of CaJAR1 was negatively correlated with the seed browning rate $(\mathrm{r}=-0.7996)$, and the expression level showed a tendency to decrease as the seed browning rate increased after chilling treatment (Figs. 4 and 6A). These results suggest that CaJAR1 is a positive regulator that influences the chilling sensitivity of pepper.

In the case of $C a E R F 1,3,5$, and 10, contrary to the expectation, there was no significant correlation with the seed browning rate after chilling (Figs. 4 and 5). In a previous study, the expression levels of CaERF 1, 3, 5 , and 10 significantly increased in the short-term chilling treatment for $24 \mathrm{~h}$, and were significantly higher in chilling-insensitive 'UZB-GJG-1999-51' pepper. However, there was no significant difference in seed browning after 3 weeks of chilling. According to previous research, in the case of the $E R F$, the expression level was high at the beginning of the chilling treatment, and decreased thereafter ${ }^{45}$. In addition, because the $E R F$ family genes are representative abiotic stress response genes, it is expected that the expression of ERFs would not be directly induced by chilling stress, but rather by damage, such as ROS generated by chilling stress, resulting in different results from previous studies.

In the case of CaERF11 and CaDREB3, which are candidate genes for negative regulators of pepper chilling response, the expression level of CaDREB3 after chilling was not significantly correlated with the seed browning rate (Fig. 4). However, the expression level of CaERF11 was highly correlated with the seed browning rate 
$(\mathrm{r}=0.7622)$, and it increased as the seed browning rate increased. Studies have shown that ERF11 promotes stem growth through the activation of gibberellin ${ }^{46}$ and suppresses the abiotic stress response of plants through antagonism with $E R F 6^{47}$. We suggest that $C a E R F 11$ is a negative regulator of the chilling response in pepper.

In conclusion, after chilling treatment, cell membrane peroxidation occurs, and ROS, including hydrogen peroxide, accumulate in the cells, and finally, seed browning of pepper occurs. In chilling-sensitive peppers, this process proceeds quickly and unlike in chilling-insensitive peppers, it proceeds slowly and weakly. Factors influencing the chilling sensitivity of pepper are expected to be regulated by the expression level of CaJAR1, which is involved in JA signaling activation, and the expression level of CaERF11, which inhibits abiotic stress response in plants.

\section{Data availability}

The original contributions presented in the study are included in the article/Supplementary Material; further inquiries can be directed to the corresponding author.

Received: 17 June 2021; Accepted: 15 February 2022

Published online: 24 February 2022

\section{References}

1. Özden, Ç. \& Bayindirli, L. Effects of combinational use of controlled atmosphere, cold storage and edible coating applications on shelf life and quality attributes of green peppers. Eur. Food Res. Technol. 214, 320-326 (2002).

2. Vámos-Vigyázó, L. \& Haard, N. F. Polyphenol oxidases and peroxidases in fruits and vegetables. Crit. Rev. Food Sci. Nutr. 15, 49-127 (1981).

3. Burton, S. G. Biocatalysis with polyphenol oxidase: A review. Cat. Today 22, 459-487 (1994).

4. Lee, J. G., Yi, G., Choi, J. H. \& Lee, E. J. Analyses of targeted/untargeted metabolites and reactive oxygen species of pepper fruits provide insights into seed browning induced by chilling. Food Chem. 332, 127406. https://doi.org/10.1016/j.foodchem.2020.127406 (2020).

5. Marangoni, A. G., Palma, T. \& Stanley, D. W. Membrane effects in postharvest physiology. Postharvest Biol. Technol. 7, 193-217 (1996).

6. Anderson, J. P. et al. Antagonistic interaction between abscisic acid and jasmonate-ethylene signaling pathways modulates defense gene expression and disease resistance in Arabidopsis. Plant Cell 16, 3460-3479 (2004).

7. Fonseca, S. et al. (+)-7-iso-Jasmonoyl-L-isoleucine is the endogenous bioactive jasmonate. Nat. Chem. Biol. 5, 344-350. https:// doi.org/10.1038/nchembio.161 (2009).

8. Hu, Y. et al. Jasmonate regulates leaf senescence and tolerance to cold stress: crosstalk with other phytohormones. J. Exp. Bot. 68, 1361-1369 (2017).

9. Hu, Y., Jiang, L., Wang, F. \& Yu, D. Jasmonate regulates the inducer of CBF expression-c-repeat binding factor/DRE binding factor 1 cascade and freezing tolerance in Arabidopsis. Plant Cell 25, 2907-2924 (2013).

10. Wang, F. et al. Phytochrome A and B function antagonistically to regulate cold tolerance via abscisic acid-dependent jasmonate signaling. Plant Physiol. 170, 459-471 (2016).

11. Seo, J., Yi, G., Lee, J. G., Choi, J. H. \& Lee, E. J. Seed browning in pepper (Capsicum annuum L.) fruit during cold storage is inhibited by methyl jasmonate or induced by methyl salicylate. Postharvest Biol. Technol. 166, 1110. https://doi.org/10.1016/j.postharvbio. $2020.111210(2020)$.

12. Koo, A. J., Gao, X., Jones, A. D. \& Howe, G. A. A rapid wound signal activates the systemic synthesis of bioactive jasmonates in Arabidopsis. Plant J. 59, 974-986 (2009).

13. Suza, W. P. \& Staswick, P. E. The role of JAR1 in jasmonoyl-L-isoleucine production during Arabidopsis wound response. Planta 227, 1221-1232 (2008).

14. Melotto, M. et al. A critical role of two positively charged amino acids in the Jas motif of Arabidopsis JAZ proteins in mediating coronatine- and jasmonoyl isoleucine-dependent interactions with the COI1 F-box protein. Plant J. 55, 979-988 (2008).

15. Mizoi, J., Shinozaki, K. \& Yamaguchi-Shinozaki, K. AP2/ERF family transcription factors in plant abiotic stress responses. BBA Gene Regul. Mech. 2, 86-96 (2012).

16. Skirycz, A. et al. Pause-and-stop: the effects of osmotic stress on cell proliferation during early leaf development in Arabidopsis and a role for ethylene signaling in cell cycle arrest. Plant Cell 23, 1876-1888 (2011).

17. Novillo, F., Medina, J. \& Salinas, J. Arabidopsis CBF1 and CBF3 have a different function than CBF2 in cold acclimation and define different gene classes in the CBF regulon. Proc. Natl Acad. Sci. USA 104, 21002-21007 (2007).

18. Sakuma, Y. et al. DNA-binding specificity of the ERF/AP2 domain of Arabidopsis DREBs, transcription factors involved in dehydration- and cold-inducible gene expression. Biochem. Biophys. Res. Commun. 290, 998-1009 (2002).

19. Jin, J. H. et al. A new ethylene-responsive factor CaPTIl gene of pepper (Capsicum annuum L.) involved in the regulation of defense response to Phytophthora capsici. Front. Plant Sci. 6, 1217 (2016).

20. Lee, J. G. et al. Jasmonic acid and ERF family genes are involved in chilling sensitivity and seed browning of pepper fruit after harvest. Sci. Rep. 10, 17949 (2020).

21. Qiao, Z. X., Huang, B. \& Liu, J. Y. Molecular cloning and functional analysis of an ERF gene from cotton (Gossypium hirsutum). BBA Gene Regul. Mech. 1779, 122-127 (2008).

22. Jin, L. G., Li, H. \& Liu, J. Y. Molecular characterization of three ethylene responsive element binding factor genes from cotton. J. Integr. Plant Biol. 52, 485-495 (2010).

23. Qi, X. N. et al. A banana fruit transcriptional repressor MaERF10 interacts with MaJAZ3 to strengthen the repression of JA biosynthetic genes involved in MeJA-mediated cold tolerance. Postharvest Biol. Technol. 120, 222-231 (2016).

24. Atkin, O. K., Loveys, B. R., Atkinson, L. J. \& Pons, T. L. Phenotypic plasticity and growth temperature: Understanding interspecific variability. J. Exp. Bot. 57, 267-281 (2006).

25. Zhou, M., Chen, H., Wei, D., Ma, H. \& Lin, J. Arabidopsis CBF3 and DELLAs positively regulate each other in response to low temperature. Sci. Rep. 7, 39819. https://doi.org/10.1038/srep39819 (2017).

26. Junglee, S., Urban, L., Sallanon, H. \& Lopez-Lauri, F. Optimized assay for hydrogen peroxide determination in plant tissue using potassium iodide. Am. J. Anal. Chem. 5, 730-736 (2014).

27. Singleton, V. L. \& Rossi, J. A. Colorimetry of total phenolics with phosphomolybdic-phosphotungstic acid reagents. Am. J. Enol. Vitic. 16, 144-158 (1965).

28. Re, R. et al. Antioxidant activity applying an improved ABTS radical cation decolorization assay. Free Radic. Biol. Med. 26, 1231-1237 (1999).

29. Park, J. H., Park, J. S. \& Choi, J. S. Basic amino acid-conjugated polyamidoamine dendrimers with enhanced gene transfection efficiency. Macromol. Res. 22, 500-508 (2014). 
30. Garcés, R. \& Mancha, M. One-step lipid extraction and fatty acid methyl esters preparation from fresh plant tissues. Anal. Biochem. 211, 139-143 (1993).

31. Livak, K. J. \& Schmittgen, T. D. Analysis of relative gene expression data using real-time quantitative PCR and the 2(-Delta Delta $\mathrm{C}(\mathrm{T})$ ) method. Methods 25, 402-408 (2001).

32. Prohens, J., Rodríguez-Burruezo, A., Raigón, M. D. \& Nuez, F. Total phenolic concentration and browning susceptibility in a collection of different varietal types and hybrids of eggplant: Implications for breeding for higher nutritional quality and reduced browning. J. Am. Soc. Hort. Sci. 132, 638-646 (2007).

33. Boonsiri, K., Ketsa, S. \& van Doorn, W. G. Seed browning of hot peppers during low temperature storage. Postharvest Biol. Technol. 45, 358-365 (2007).

34. Yu, M., Gouvinhas, I., Rocha, J. \& Barros, A. Phytochemical and antioxidant analysis of medicinal and food plants towards bioactive food and pharmaceutical resources. Sci Rep. 11, 10041. https://doi.org/10.1038/s41598-021-89437-4 (2021).

35. Thorpe, G. W. et al. Superoxide radicals have a protective role during $\mathrm{H}_{2} \mathrm{O}_{2}$ stress. Mol. Biol. Cell 24, 2876-2884 (2013).

36. Das, K. \& Roychoudhury, A. Reactive oxygen species (ROS) and response of antioxidants as ROS-scavengers during environmental stress in plants. Front. Environ. Sci. 2, 53 (2014).

37. Duan, J. et al. OsMIOX, a myo-inositol oxygenase gene, improves drought tolerance through scavenging of reactive oxygen species in rice (Oryza sativa L.). Plant Sci. 196, 143-151 (2012).

38. Prabakaran, M. et al. Effect of storage time and temperature on phenolic compounds of soybean (Glycine max L.) flour. Molecules 23, 2269 (2018).

39. Faramarz, S., Dehghan, G. \& Jahanban-Esfahlan, A. Antioxidants in different parts of oleaster as a function of genotype. BioImpacts 5, 79 (2015).

40. La Torre, C. et al. Effects of long-term storage on radical scavenging properties and phenolic content of kombucha from black tea. Molecules 26, 5474 (2021).

41. Farmer, E. E. \& Mueller, M. J. ROS-mediated lipid peroxidation and RES-activated signaling. Annu. Rev. Plant Biol. 64, 429-450 (2013).

42. Kinnersley, A. M. \& Turano, F. J. Gamma aminobutyric acid (GABA) and plant responses to stress. Crit. Rev. Plant Sci. 19, 479-509 (2000).

43. Binder, S. Branched-chain amino acid metabolism in Arabidopsis thaliana. Arabidopsis Book (American Society of Plant Biologists) 8, e0137; https://doi.org/10.1199/tab.0137 (2010).

44. Chini, A. et al. The JAZ family of repressors is the missing link in jasmonate signalling. Nature 448, 666-671 (2007).

45. Fan, W. et al. The ERF transcription factor family in cassava: Genome-wide characterization and expression analyses against drought stress. Sci. Rep. 6, 37379. https://doi.org/10.1038/srep37379 (2016).

46. Zhou, X. et al. The ERF11 transcription factor promotes internode elongation by activating gibberellin biosynthesis and signaling. Plant Physiol. 171, 2760-2770 (2016).

47. Dubois, M. et al. The ETHYLENE RESPONSE FACTORs ERF6 and ERF11 antagonistically regulate mannitol-induced growth inhibition in Arabidopsis. Plant Physiol. 169, 166-179 (2015).

\section{Acknowledgements}

We thank the members of the Horticultural Crop Molecular Physiology Lab for their assistance in preparing the plant materials.

\section{Author contributions}

Study concepts: J.G.L., E. J. L., Study design: J.G.L., E.J.L., Data acquisition and analysis: J.G.L., J.S., Manuscript preparation: J.G.L., E.J.L., manuscript editing and review: B.C.K., J.H.C., and E.J.L.

\section{Funding}

This work was supported by the Basic Science Research Program through the National Research Foundation (NRF, 2020R1F1A1068842 to E.J.L.) of Republic of Korea, funded by the Ministry of Education, Science, and Technology, the Korea Institute of Planning and Evaluation for Technology in Food, Agriculture, and Forestry (IPET, 617068-05-1-WT111 to E.J.L.), and the Rural Development Administration (RDA, grant number PJ01600502), Republic of Korea.

\section{Competing interests}

The authors declare no competing interests.

\section{Additional information}

Supplementary Information The online version contains supplementary material available at https://doi.org/ 10.1038/s41598-022-07268-3.

Correspondence and requests for materials should be addressed to E.J.L.

Reprints and permissions information is available at www.nature.com/reprints.

Publisher's note Springer Nature remains neutral with regard to jurisdictional claims in published maps and institutional affiliations.

(c) (i) Open Access This article is licensed under a Creative Commons Attribution 4.0 International License, which permits use, sharing, adaptation, distribution and reproduction in any medium or format, as long as you give appropriate credit to the original author(s) and the source, provide a link to the Creative Commons licence, and indicate if changes were made. The images or other third party material in this article are included in the article's Creative Commons licence, unless indicated otherwise in a credit line to the material. If material is not included in the article's Creative Commons licence and your intended use is not permitted by statutory regulation or exceeds the permitted use, you will need to obtain permission directly from the copyright holder. To view a copy of this licence, visit http://creativecommons.org/licenses/by/4.0/.

(C) The Author(s) 2022 